\title{
Les nombreuses vies de l'art. 47c LAMal
}

\section{Yvonne Gilli}

Dre méd., présidente de la FMH

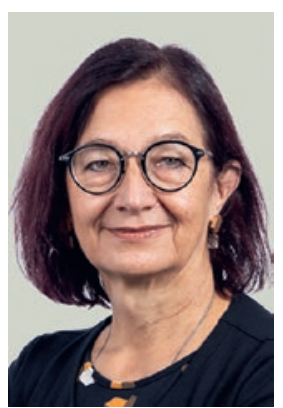

Celles et ceux qui suivent attentivement l'actualité politique ont régulièrement des impressions de déjà-vu. Sauf qu'en politique, le déjà-vu est à prendre au sens strict, c'est-à-dire qu'il ne s'agit pas de la sensation troublante de revivre une scène mais du souvenir bien réel d'un projet politique qui a déjà été discuté plusieurs fois (et rejeté).

C'est notamment le cas ces jours-ci alors que le Parlement se penche une nouvelle fois sur l'ajout d'un article 47c dans la LAMal, un projet de loi qui aurait un énorme impact sur notre système de santé. L'article exige en effet des «règles pour corriger les augmentations injustifiées des volumes et des coûts». Ce qui au premier abord peut paraître anodin jette de facto les jalons d'une limite supérieure jusqu'à laquelle les traitements seraient considérés comme "justifiés». Lorsque ce plafond est dépassé, les médecins s'exposent à des sanctions financières. Nous serions pour ainsi dire contraints de ne pas aller au-delà d'un seuil de coûts prédéfini, indépendamment de ce dont ont besoin nos patientes et nos patients.

Rien de surprenant si ces exigences vous semblent étrangement familières: en 2017 déjà, l'initiative de la commission 17.402, dont la teneur était en partie similaire, exigeait également une enveloppe budgétaire. En pointant les coûts de la santé, la commission de la santé

\section{Le souvenir qu'un projet politique a déjà été} discuté plusieurs fois (et rejeté) est bien réel.

\section{Références} Liste complète des références sous www.bullmed.ch ou via code $Q R$

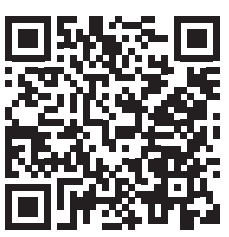

du Conseil national (CSSS-N) faisait en mai 2018 vaillamment la promotion de cette initiative qui devait soutenir le Conseil fédéral dans ses efforts pour imposer des mesures visant à maîtriser la hausse des coûts [1]. Après une discussion de fond, notamment sur le rationnement des soins que pourrait engendrer le plafonnement des coûts, le Conseil national a finalement changé d'avis et torpillé l'initiative par 97 voix contre 91 [1].

Avec cette défaite parlementaire, les «mesures des partenaires tarifaires concernant le pilotage des coûts» auraient sans doute été de l'histoire ancienne si le Département fédéral de l'intérieur (DFI) ne leur avait pas donné une seconde vie: le premier volet mis en consultation à l'automne 2018 comprenait huit mesures découlant du rapport d'experts, dont l'article 47 c, pour répondre au souhait du DFI [2]. Initialement passée à trappe, l'initiative 17.402 était remise en selle par le rapport d'experts et retrouvait ainsi sa place dans le volet de mesures adopté par le Conseil fédéral en 2019.

\section{Il serait regrettable qu'un tel impact sur} le système de santé soit imposé à la faveur de majorités extrêmement courtes.

S'en sont suivis de nombreux votes au Parlement, qui n'auraient pas pu être plus serrés. En août 2020, la commission de la santé du Conseil national a décidé par 12 voix contre 11 de ne pas biffer l'article $47 \mathrm{c}$ tandis que le Conseil national, quelques mois plus tard, décidait de le biffer par 91 voix contre 90. Un an plus tard, en octobre 2021, la commission de la santé du Conseil des Etats décidait à son tour, par 7 voix contre 6, de ne pas le biffer et, quelques mois plus tard, en décembre 2021, le Conseil des Etats prenait la décision de le biffer par 21 voix contre 20. Ces deux votes au Conseil national et au Conseil des Etats semblaient avoir mis définitivement un terme à la deuxième vie de l'article $47 \mathrm{c}$.

Pourtant, l'usage n'a pas l'air d'effrayer l'article 47 c: en janvier 2022, les propositions de réexamen déposées dans les deux commissions de la santé lui ont redonné une troisième vie. On ne peut que supposer que le DFI a de nouveau joué un rôle en arrière-plan. Au jeu des décisions prises de justesse, la CSSS-N a finalement opté pour la suppression de l'article $47 \mathrm{c}$, cette fois avec l'aide de la voix prépondérante de son président, et il ne reste plus qu'à espérer que le Conseil national la suivra le 28 février prochain. Il serait regrettable que l'impact massif de l'article $47 \mathrm{c}$ sur notre système de santé nous soit imposé avec insistance et quelques manœuvres à la faveur de majorités extrêmement courtes. D’autant plus que le prochain déjà-vu se prépare. Avec le contreprojet indirect pour un «objectif de maîtrise des coûts", nous devrons très bientôt discuter de nouveau d'un budget pour la santé. 